\title{
Electrochemical Noise Chaotic Analysis of NiCoAg Alloy in Hank Solution
}

\author{
D. Bahena, I. Rosales, O. Sarmiento, R. Guardián, C. Menchaca, and J. Uruchurtu
}

Centro de Investigación en Ingeniería y Ciencias Aplicadas, UAEM Avenida Universidad 1001, Col. Chamilpa, Cuernavaca, MOR, Mexico

Correspondence should be addressed to J. Uruchurtu, juch25@uaem.mx

Received 27 January 2011; Accepted 30 March 2011

Academic Editor: Ravin Kumar Dayal

Copyright ( $\odot 2011$ D. Bahena et al. This is an open access article distributed under the Creative Commons Attribution License, which permits unrestricted use, distribution, and reproduction in any medium, provided the original work is properly cited.

The potential and current oscillations during corrosion of NiCoAg alloy in Hank solution were studied. Detailed nonlinear fractal analyses were used to characterize complex time series clearly showing that the irregularity in these time series corresponds to deterministic chaos rather than to random noise. The chaotic oscillations were characterized by power spectral densities, phase space, and Lyapunov exponents. Electrochemical impedance was also applied the fractal dimensions for the corroded surface was obtained, and a corrosion mechanism was proposed.

\section{Introduction}

The wide diversity and sophistication of materials currently used in medicine and biotechnology are a testimony to the significant technological advances which have occurred over the past 25 years; metallic implant materials have a significant economic and clinical impact on the biomaterials field. The demand for such medical device implants is expected to increase in the coming years. Stainless steels, cobalt, titanium and their alloys are widely used in the production of artificial joints and fixation devices where both titanium, and cobalt alloys demonstrate good biocompatibility. Many other alloys are being developed to improve performance and to lower their costs $[1,2]$. For such new materials little is known about their electrochemical characteristics and dynamic corrosion behaviour.

Spontaneous electrochemical potential and current oscillations generated by corrosion reactions are known as electrochemical noise (EN). These oscillations are commonly observed in many electrochemical processes, such as electropolishing, passivation, active and different forms of localized corrosion, which are strongly influenced by the growth and the dynamic film breakdown and repassivation events of the anodic film.

For the interpretation of electrochemical noise measurements (ENMs) data, different approaches have been pro- posed for processing the experimental data records: visual, statistical, spectral, and wavelet transform-based analysis, and the less common chaos-fractal non-linear dynamic systems analysis. Reviews of the measurements interpretation of electrochemcal noise describing these analysis and their practical applications associated with corroding metals can be found in the literature [3-6]. Cottis [6] questions the usefulness of chaos theory regarding electrochemical noise analysis. Legat et al. $[7,8]$, and others carrying out chaotic analysis of electrochemical noise in various corrosion processes, concluded that the chaotic analysis of measured electrochemical noise can help to determine the different types of corrosion [8-24].

Interest in the behavior of nonlinear dynamic systems has considerably increased. Under certain circumstances, these systems exhibit a behavior that has been termed "deterministic chaos." This means that the system behaves in a deterministic way (i.e., stochastic, but not random); however, its behavior is extremely sensitive to the initial conditions, therefore being in the long range completely unpredictable.

In the last few years, chaos theory has been developed to characterize nonperiodic and non-linear systems. This theory has been applied in the characterization of various systems, including electrochemical processes. The application of deterministic chaos theory to materials science reveals 
the prevalence of nonlinear dynamic effects on the behavior of materials.

Pitting may exhibit either random or deterministic (sometimes chaotic) features following the composition of the corrosive electrolyte with respect to the pitting resistance of the material under consideration. The pitting corrosion of copper in artificial seawater is chaotic in nature as well as the pitting process of aluminum and nickel at its free corrosion potential in the presence of chlorides $[10,15,20,21,25]$.

Electrochemical noise (EN) is a nonintrusive technique used which is highly successful as a powerful tool both for the fundamental understanding of various aspects of corrosion and for the solution of practical problems of corrosion protection, in particular in the area of corrosion monitoring. The high sensitivity of the technique also permits detection of the initiation of localized corrosion on passive metal surfaces [3-5].

The aim of the present study was to analyze the EN measured on a nickel cobalt silver alloy developed as a possible biomaterial immersed in Hank solution. By the use of different chaos and fractal analysis tools, the dynamic behavior and the mechanism of different types of corrosion were characterized and compared with other electrochemical techniques.

\section{Experimental Procedure}

The Ni-Co-Ag alloy was produced by the Vacuum Induction Melting (VIM) technique, in a range of system pressure of $10^{-2}$ torr. The alloy was melted in high-purity quartz crucibles of $13 \mathrm{~mm}$ diameter with $\mathrm{Ni}$ content of $60 \mathrm{wt} . \%$, Co 35 wt. $\%$, and Ag content 5 wt.\%. Heat treatment was performed at $850^{\circ} \mathrm{C}$ for one hour in order to homogeneize the microstructure of the alloy. Metal specimens leaving an exposed area of $1.0 \mathrm{~cm}^{2}$ were encapsulated in epoxy resin and polished with silicon carbide ( $\mathrm{SiC}$ ) paper up to 1200 grit, and with diamond paste to a $1.0 \mu \mathrm{m}$ finish. Samples were washed with distilled water, degreased with acetone, dried under an air stream, and kept in a desiccator until immersion.

The sample was used as a working electrode in a typical electrochemical cell using a four-electrode setup including the two identical working electrodes, a saturated calomel reference electrode (SCE) and a graphite counter electrode, for other electrochemical measurement techniques. Electrochemical experiments were performed using an ACM Electrochemical Instrument controlled by a personal computer. The electrolyte was a Hank solution whose composition is given in Table 1.

The ENM time series were carried out using an ACM Electrochemical Instrument coupled to a personal computer (PC), which could store the data for further analysis. The method consisted of measuring as a function of time, the electrochemical potential noise (EPN) between the working electrode and a SCE, and the electrochemical current noise (ECN) between two identical working electrodes, at open circuit conditions. The 4096 data samples were obtained at a sampling rate of one reading every 1 second, controlled by a PC. After gathering the data, four segments of 1024 data samples were produced and averaged, obtained, and presented
TABLE 1: Hank solution composition.

\begin{tabular}{lc}
\hline Component & Concentration $(\mathrm{g} / \mathrm{L})$ \\
\hline $\mathrm{NaCl}$ & 8.0 \\
$\mathrm{CaCl}_{2}$ & 0.14 \\
$\mathrm{KCl}$ & 0.4 \\
Glucose & 1.0 \\
$\mathrm{NaHCO}_{3}$ & 0.35 \\
$\mathrm{MgCl}_{2} \cdot 6 \mathrm{H}_{2} \mathrm{O}$ & 1.0 \\
$\mathrm{NaHPO}_{4} \cdot 2 \mathrm{H}_{2} \mathrm{O}$ & 0.06 \\
$\mathrm{KH}_{2} \mathrm{PO}_{4}$ & 0.06 \\
$\mathrm{MgSO}_{4} \cdot 7 \mathrm{H}_{2} \mathrm{O}$ & 0.06 \\
\hline
\end{tabular}

as a potential/current time record of sufficient length, 1024 data samples, and the direct current (DC) drift was removed when necessary, for further analysis. This trend removal is a common procedure in electrochemical noise measurements procedure and analysis, since the DC drift overshadows the properties and magnitude of true electrochemical noise. Electrochemical noise analysis was performed using the chaos data analyzer educational software.

Electrochemical impedance spectroscopy tests, EIS, were carried out at $E_{\text {corr }}$ by using a signal with an amplitude of $10 \mathrm{mV}$ and a frequency interval of $1.00 \mathrm{E}+4$ to $1.00 \mathrm{E}-2 \mathrm{~Hz}$ every 24 hours during 30 days. Experiments were performed in static conditions at $37^{\circ} \mathrm{C}$ by immersing $1000 \mathrm{~mL}$ electrochemical cell in a thermostatic bath. Afterwards, analysis was performed with suitable impedance analysis software by using an equivalent circuit.

Potentiodynamic polarization curves were obtained by varying the applied potential from $-1000 \mathrm{mV}$ up to $+1000 \mathrm{mV}$ at a scan rate of $60 \mathrm{mV} / \mathrm{s}$. Before the experiments, the $E_{\text {corr }}$ value was measured during approximately $30 \mathrm{~min}$ utes, until reaching stability. All potentials were measured using a saturated calomel electrode (SCE) as a reference electrode. All tests were performed at $37 \pm 1^{\circ} \mathrm{C}$.

\section{Results and Discussion}

Electrochemical potential (EPN) and current (ECN) noise were measured in a freely corroding system (see Figure 1), to obtain information regarding the corrosion process. The NiCoAg alloy in the Hank solution electrolyte suffers general and/or localized corrosion. In general, three different types of EN generated by different types of corrosion were observed. Type A (Figure 1 [potential Day 1] and [current Day 1]): the EN generated consisted of high-frequency (high repetition rate) oscillations and high-amplitude trains of modulated amplitude oscillations with a low-frequency quasiperiodic oscillation. Type B (Figure 1 [potential Day 12] and [current Day 12]): oscillations of type A combined with occasional high-amplitude sudden transients and type C (Figure 1 [potential Day 30] and [current Day 30]) formed by small amplitude oscillations with some higher amplitude transients.

The behaviour observed with time series of different shapes seems to indicate that the corrosion products formed 

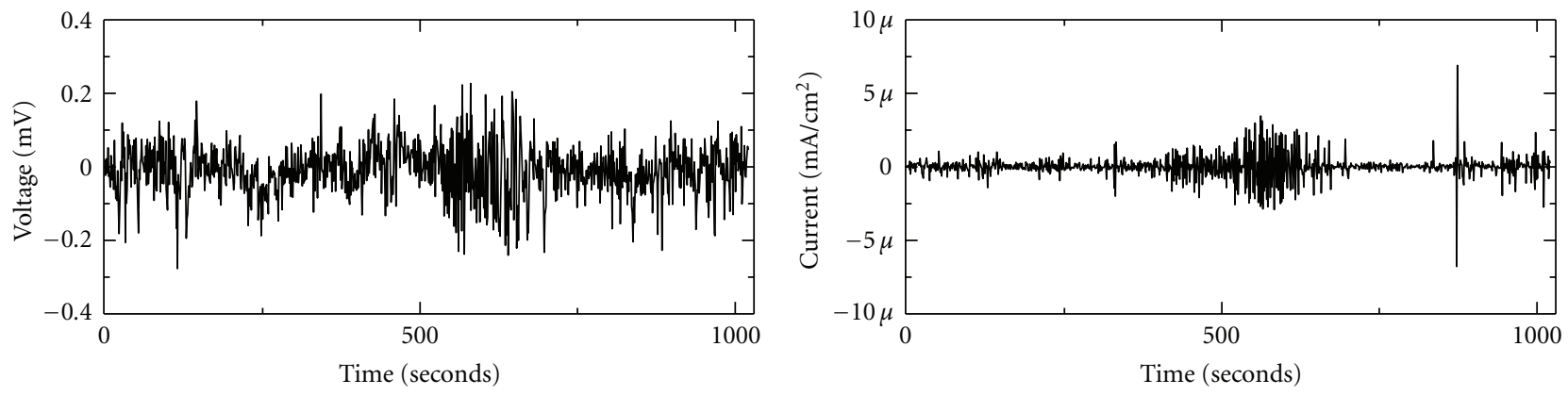

- Day 1
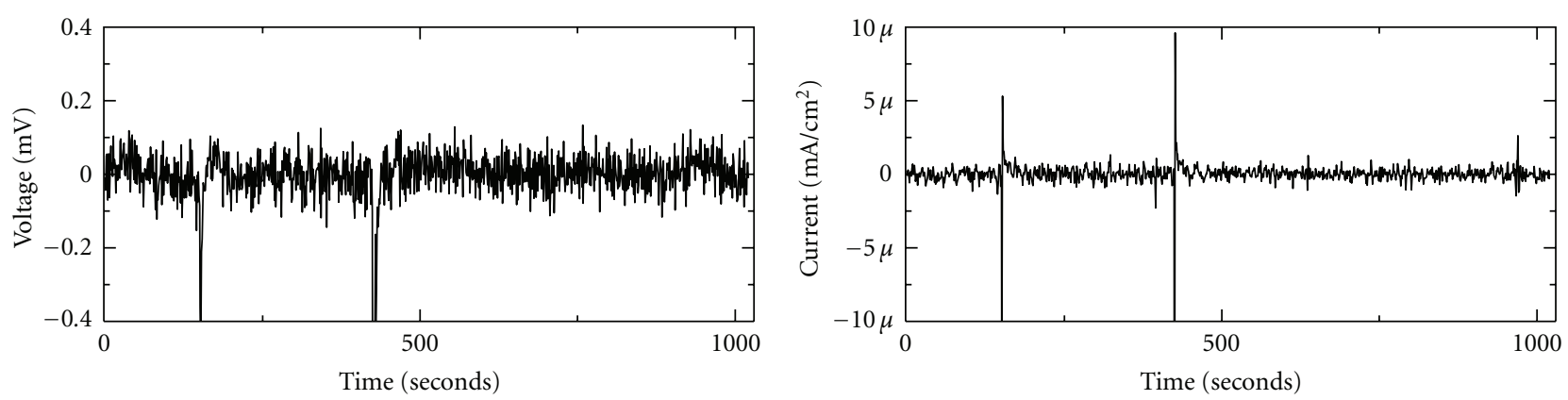

Day 12
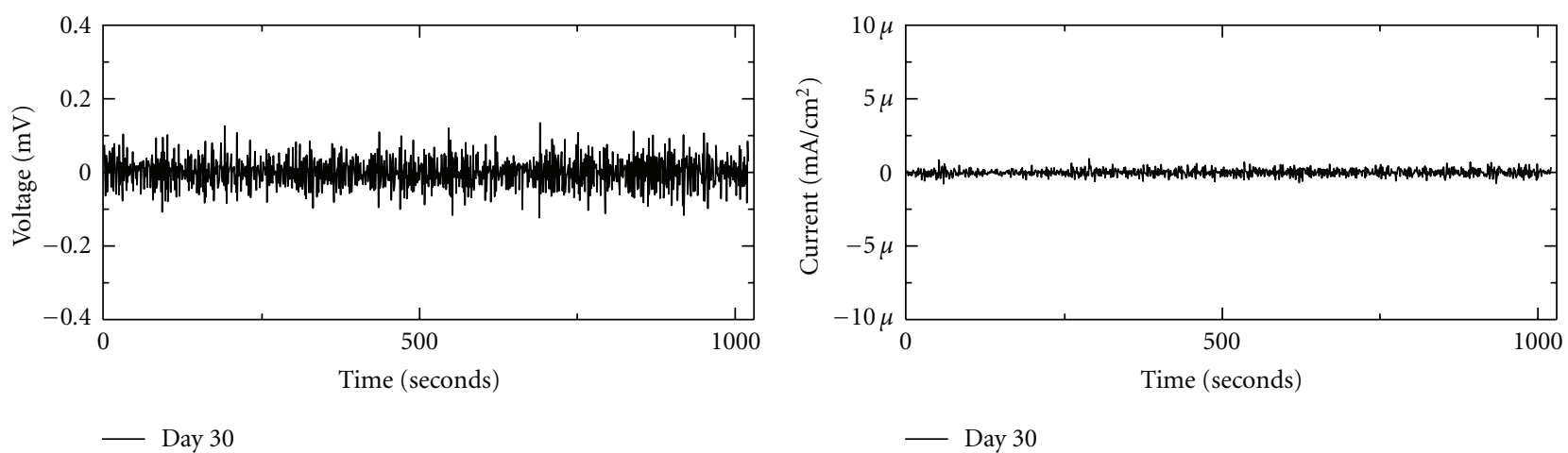

(a)

(b)

FIGURE 1: Electrochemical potential and current time series for different immersion.

over the metal surface have a different composition and degree of stability (EPN), thus producing a corrosive attack whose kinetics and morphology are different (ECN). The EN of type A is associated with pitting corrosion (Figure 2(a)), whereas EN type $C$ signals seem to correspond to general corrosion or achievement of a pseudopassivation (corrosion products) state (Figure 2(b)). Type B could be ascribed to a possible transition state between the two.

This fact is also reflected in the changing corrosion potential values as a function of time and the polarization curve (Figure $3(\mathrm{a})$ ). The potential as a function of time presents a trend towards more active values. Nevertheles, three distinctive regions can be observed, the first few days oscillating potentials, from the tenth to the twentieth day less variable potentials with a trend to decrease in their values, from the twentieth day onwards, steady state values were observed. This was due to the possible formation of protective corrosion products but maintaining active corrosion potential values. It was confirmed by the polarization curve (Figure 3(b)), where the anodic region presents an anodic pseudopassive region associated to the formation of a protective film and a pitting potential. The cathodic region presents a typical limit current behavior associated to mass transfer process responsible for the mixed control of the corrosion process.

Spectral analysis has also been used to study the periodicity of the structure of EN time records. The slope of the spectral density function (SDF) at higher frequencies typically has the form of $\left(1 / f^{\beta}\right)$. Different values of the $\beta$ exponent have been reported for specific modes of corrosion [10, 26-28]. Mandelbrot provides the connection between the structure of the EN time record and the SDF 


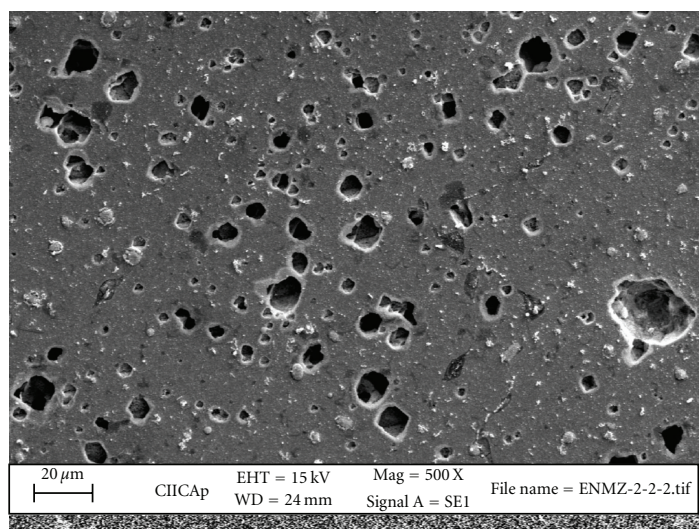

(a) Pitting

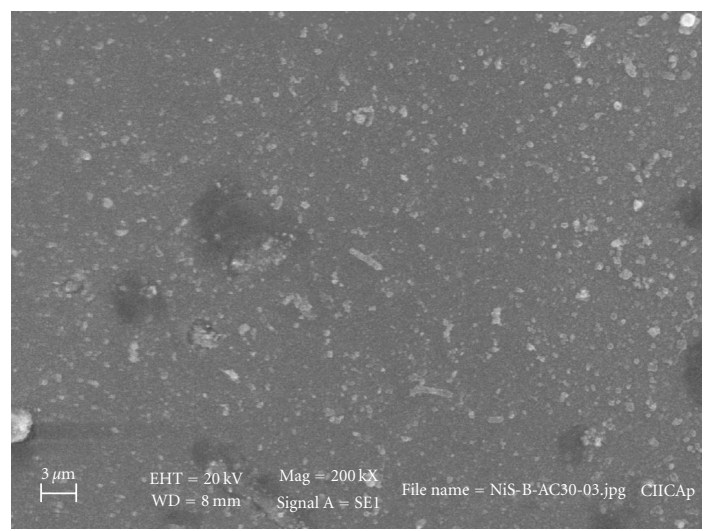

(b) Pseudo passivation

FIGURE 2: SEM micrograph showing morphology attack.

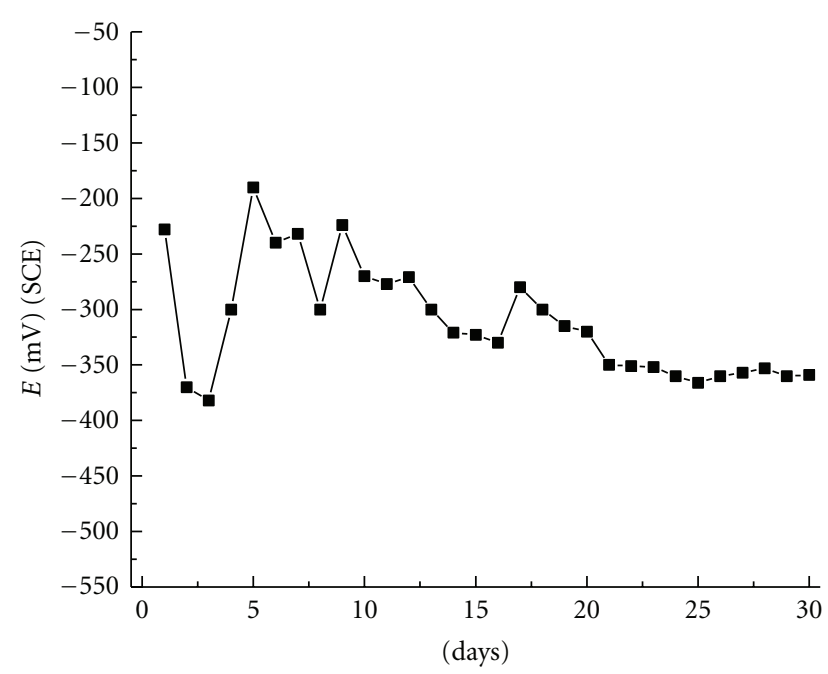

(a) Potential

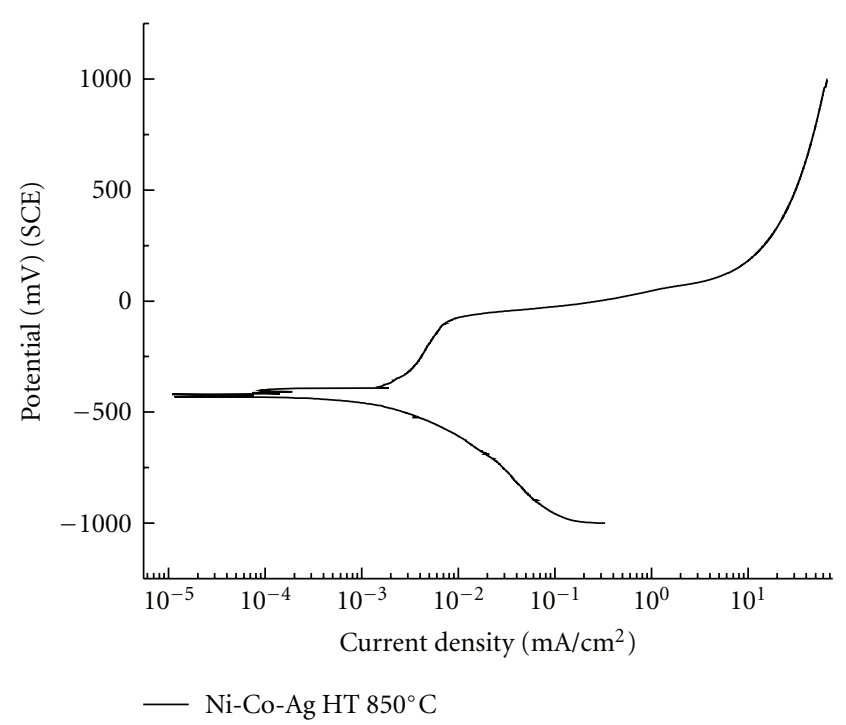

(b) Polarization

FIgURE 3: Corrosion potential as a function of time and polarization curve.

(characterized by $D_{f}$ and $\beta$ ) and the microscopic behaviour (oxidation reactions) responsible for corrosion. The fractal dimension $D_{f}$ is defined as

$$
D_{f}=\frac{(5-\beta)}{2} \text {. }
$$

The power spectrum based on the discrete Fast Fourier Transform (FFT) refers to the transformation of functions of a continuous real argument, such as time $(t)$. The Fourier transform describes a function $f(t)$ in terms of basic complex exponentials of various frequencies. In terms of ordinary frequency, $v$, the Fourier transform is given by the complex number through the following:

$$
F(\nu)=\int_{-\infty}^{\infty} f(t) \cdot e^{-i 2 \pi v t} d t
$$

Evaluating this quantity for all values of frequency $v$ produces the frequency-domain function. The power spectrum, which is a graph of the amplitude spectral density against frequency of voltage and current noise corresponding to EN Types $A$ and $C$, is shown in Figure 4. Two types of behaviour were observed in the spectra obtained, white noise which is independent of frequency and the other a $1 / f^{\beta}$ function. When localized attack is the dominant mechanism like in pitting corrosion process, the EN signal time series present high-frequency transients of increasing amplitude, according to Uruchurtu and Dawson [10]. Localized corrosion of the metal alloy tested was observed in Hank solution (Figure 3).

The EN obtained for localized corrosion, being of type A, can be presumed to have been generated by galvanic corrosion initiating localized corrosion attack around silver particles. These particles eventually become loose and precipitate leaving active sites which eventually are covered 

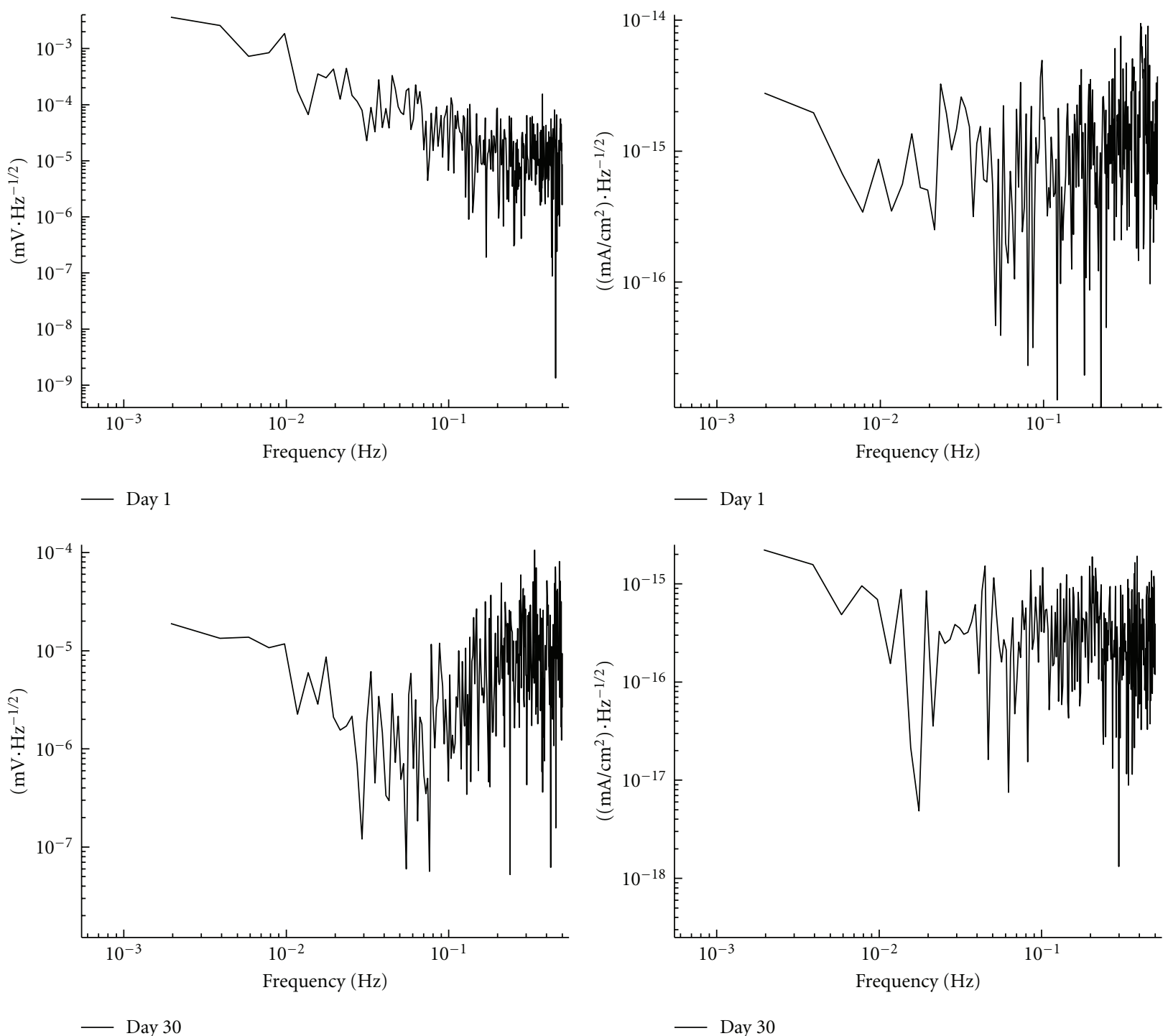

(a) EN potential

(b) EN current

FIgURE 4: EN spectral density plots for 1 and 30 days of immersion.

or stiffened with corrosion products. This process might be responsible for the train of pulses or bursts of noise observed (types A and C). These particles eventually become loose and precipitate leaving active sites which eventually are covered or stiffened with corrosion products. Other sites corrode and are covered with corrosion products film [25].

As an example, two types of spectra (Figure 4) were obtained for type A and B. The amplitude spectra of voltage and current noise show that the low-frequency limit components changed from $2 \times 10^{-5} \mathrm{mV} / \mathrm{Hz}^{1 / 2}$ to $4 \times$ $10^{-3} \mathrm{mV} / \mathrm{Hz}^{1 / 2}$ for voltage and $1 \times 10^{-15} \mathrm{~mA} / \mathrm{cm}^{2} / \mathrm{Hz}^{1 / 2}$ to $3 \times 10^{-15} \mathrm{~mA} / \mathrm{cm}^{2} / \mathrm{Hz}^{1 / 2}$ for current noise, respectively. The amplitude values, at $\sim 500 \mathrm{mHz}$ high frequency limit, increase from $1 \times 10^{-5} \mathrm{mV} / \mathrm{Hz}^{1 / 2}$ to $5 \times 10^{-5} \mathrm{mV} / \mathrm{Hz}^{1 / 2}$ for voltage. This change could be associated with the change from type A to type C signals. Analogously, the current noise increases from $2 \times 10^{-16} \mathrm{~mA} / \mathrm{cm}^{2} / \mathrm{Hz}^{1 / 2}$ to $1 \times$ $10^{-15} \mathrm{~mA} / \mathrm{cm}^{2} / \mathrm{Hz}^{1 / 2}$, for the same reasons. This type of spectrum has been attributed to chaotic signals due to the $1 / f^{\beta}$ shape obtained. Chaotic analysis, therefore, has been applied to characterize the processes generating the different types of EN [7].

All phenomena of high-amplitude oscillations can be associated with the possible formation of unstable films, which are likely formed of nickel and cobalt oxides and/or chlorides [27, 28]. The stabilization of these layers can take place through the formation of complex species of a salt film, as indicated by García et al. [15] for nickel in chlorides. The presence of complex species, which may change the stability of corrosion films, could be an explanation for the different noise signals obtained. This is confirmed by the shape of the polarization curve (Figure 3(b)), where a paqssive region of 


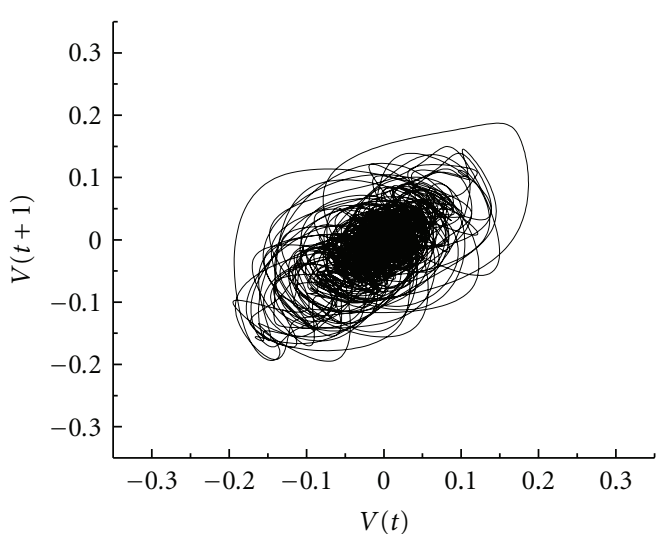

- Day 1

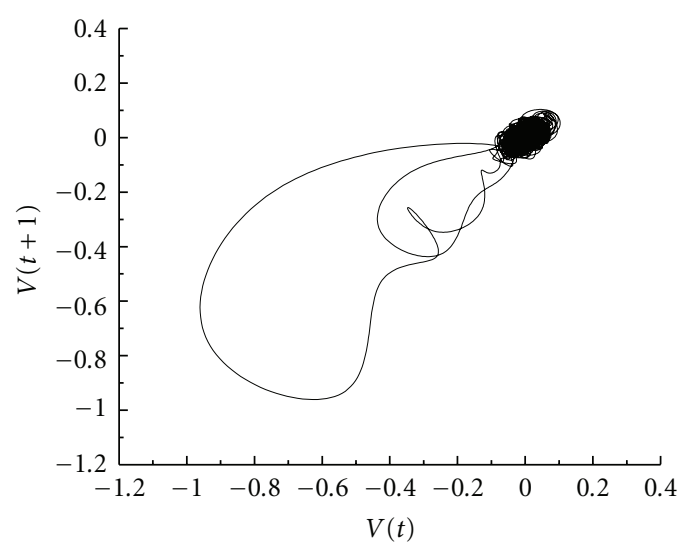

— Day 12

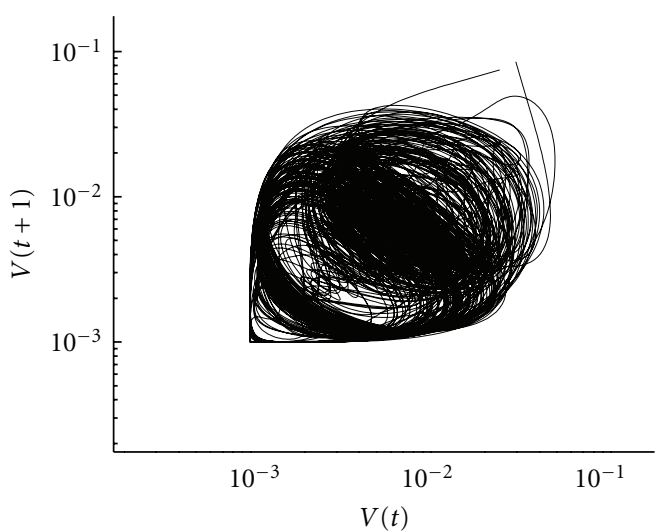

— Day 30

(a) EN potential

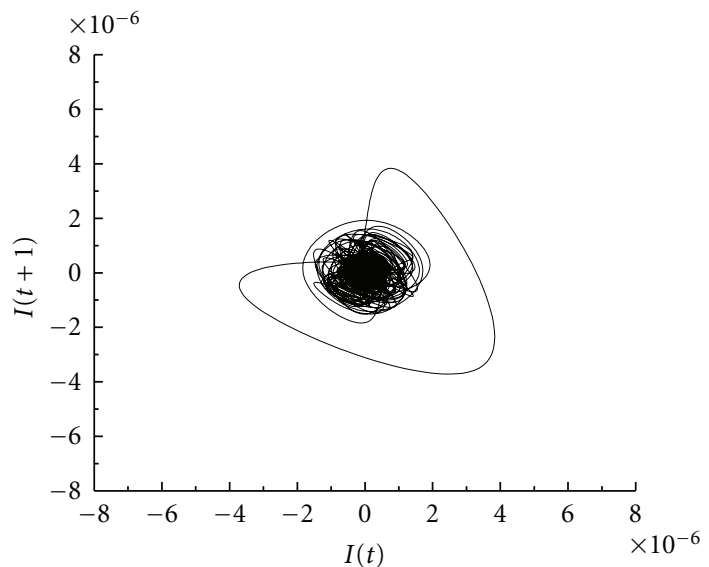

Day 1

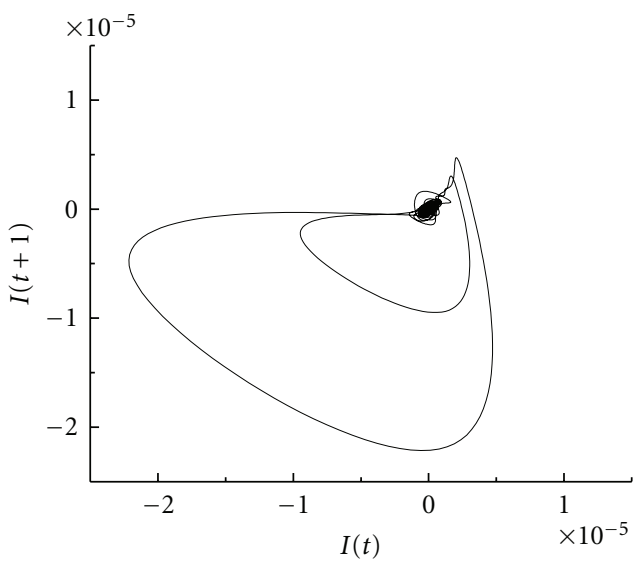

- Day 12

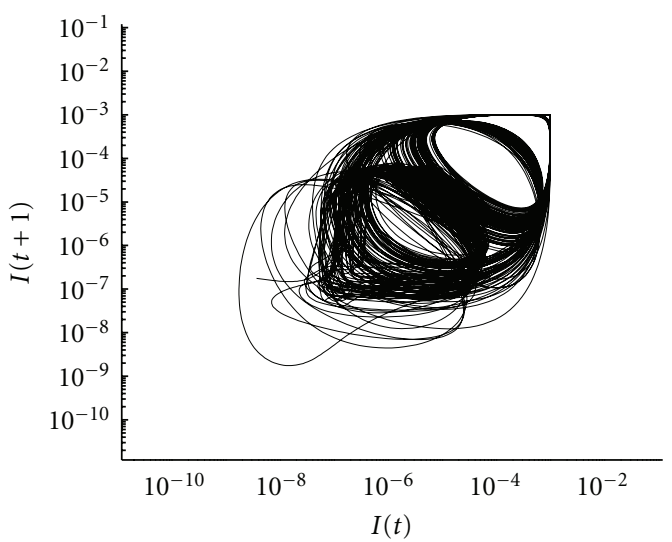

Day 30

(b) EN current

FIgURE 5: EN attractors for different times of immersion.

about $200 \mathrm{mV}$ associated to the corrosion product formation is present, and a pitting potential around $-150 \mathrm{mV}$. The corrosion potential is around $-350 \mathrm{mV}$ and a current limit cathodic region associated to diffusion process.

It is possible to distinguish a stochastic (random) behaviour from a deterministic one. The behavior of a dynamic system is best described in its phase space portrait, a graph of the variable $X(t)$, against its time derivative series. Each possible state of the system corresponds to a point in the phase system, whereas the evolution of states is represented by a continuous line giving the system trajectory. If the signal is purely stochastic, the trajectory fills randomly the 


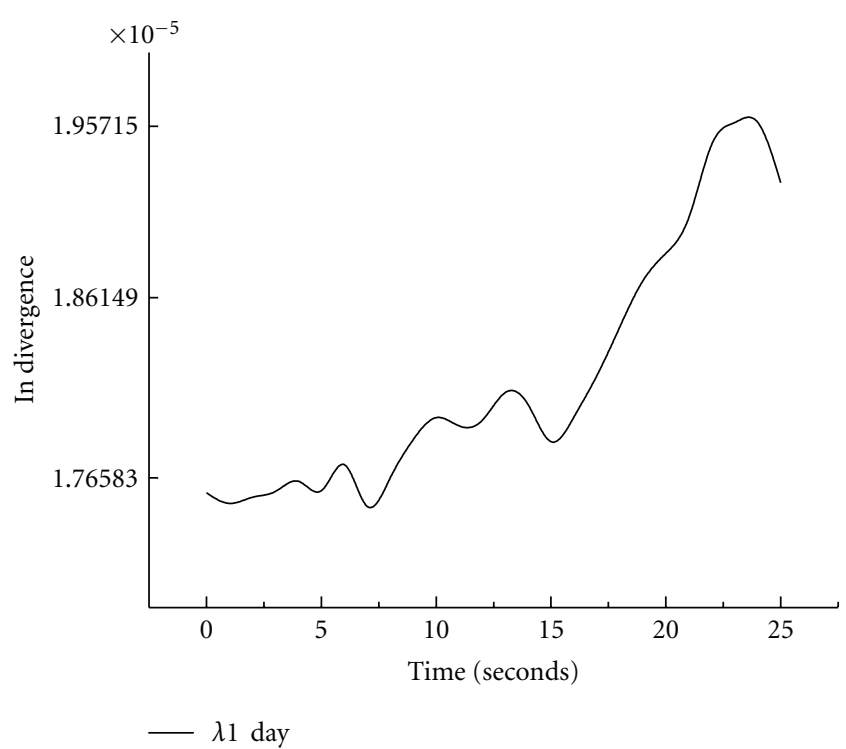

FIGURE 6: Average divergence for the Lyapunov exponent.

phase space. A graph describing the evolution of the system from different initial conditions is called an attractor. When the signal is deterministic, the trajectory draws a particular shape, whose topology determines the level of organization for the system behaviour.

Even when the system seems to obey deterministic law (in probabilistic terms), the long-term behaviour may be unpredictable, because of its sensitivity to the initial conditions. This sensitivity characterizes the onset of lowdimensional chaos, together with self-similarity properties of the attractor which then exhibit a fractal structure [29]. From the latter, it is evident that different types of EN form different types of attractors [17-19].

Graphic presentations of attractors for the different potential and current noise signals obtained shown in Figure 5. During localized corrosion (EN type A, day 1), the attractor consisted of multiple loops, and a diamond ringlike attractor for current (Figures 5(a) [day 1] and 5(b) [day 1]) was obtained. The attractors of EN generated by general and mixed corrosion (EN type B, day 12) indicated a transition state from high to low periodicity and are clearly distinguished (Figures 5(a) [day 12] and 5(b) [day 12]). During passivation (EN type C, day 30), the attractor of voltage and current noise (Figures 5(a) [day 30] and 5(b) [day 30]) presents a cone-like attractor and low periodicity.

The attractors of different types of EN form different structures; however, to quantify the geometry of an attractor, its static and dynamic characteristics (Lyapunov exponents) must be estimated. The Lyapunov exponents are the most important parameters characterizing the properties of an attractor of a dynamical system. The Lyapunov exponents measure the average rates of divergence of nearby trajectories in phase space, thus quantifying how unpredictable the system is, depending on initial conditions. When at least one Lyapunov exponent is positive, then this system exhibits chaotic behaviour. On the other hand when no positive
Lyapunov exponent exists, the long-term predictability of the system is guaranteed.

In order to characterize the unpredictability of processes that generate different types of EN, the maximum Lyapunov exponent has to be estimated. To do this, a method was proposed by Rosenstein et al. [30] and commonly used. The maximum Lyapunov exponent, $\lambda$, quantifies the greatest rate at which the distance between two nearby trajectories increases exponentially in time. If $\lambda>0$, there is at least one direction in the phase space along which an attractor exhibits chaotic (unstable) behavior; for $\lambda=0$, some sort of steady state do exist $[31,32]$.

To calculate the Lyapunov exponents an algorithm such as the following can be used:

$$
\lambda=\lim _{N \rightarrow \infty} \frac{1}{N} \sum_{n=1}^{N} \log \left|\frac{d x_{n+1}}{d x_{n}}\right|=\lim _{N \rightarrow \infty} \frac{1}{N} \sum_{n=1}^{N} \log \left|r_{n}\left(1-2 x_{n}\right)\right| .
$$

Quantitatively, two trajectories in phase space with initial separation $\delta Z_{0}$ diverge (provided that the divergence can be treated within the linarization approximation), therefore, the divergence can be obtained by

$$
|\delta Z(t)| \approx e^{\lambda t}\left|\delta Z_{0}\right|
$$

As an example Figure 6 presents the average divergence for the Lyapunov exponent, obtained for the current noise phase space attractor, corresponding to the first day of immersion. The values of the $\lambda 1$ estimated for the attractors of electrochemical potential and current noise in the different types of corrosion were calculated over a period of 30 days (Figure 7 ). It can be seen that the positive values of $\lambda$ oscillate near or above zero. In general, at the start they are lower than 0.2 for EN type A, whereas for the mid-period the values of $\lambda$ were between 0.2 and 0.4 for EN type $B$ and at the end were higher than 0.4 for EN type C. Three regions can be distinguished in this plot: from 0 to 15 days, with alternation between EN type A and B; from 15 to 22 days, with values of $\lambda 1$ lower than 0.2 , corresponding to EN type B; from 23 to 30 days, with values of $\lambda 1$ higher than 0.4 (EN type $C$ ), which is in accordance with Figure 3(b), corresponding to general corrosion or pseudo passivation.

It can be noted that during localized corrosion (EN type A), the $\lambda 1$ is positive, which is in accordance with the results previously published by Corcoran and Sieradzki [18], demonstrating that the fluctuations that occur during this process are chaotic in nature.

The electrochemical impedance data as a function of time, of the alloy immersed in Hank solution, is presented in Figure 8 [33-36]. The results show the Nyquist diagrams describing the different behavior of the alloy for different times of immersion in the corrosive medium. It is seen that there is present single depressed semicircle involving one time constant $(\tau)$.

Alternatively, for longer periods of immersion, small and increasingly large depressed semicircles, at low frequency corresponding to mass transfer adsorption process, were sometimes obtained. 


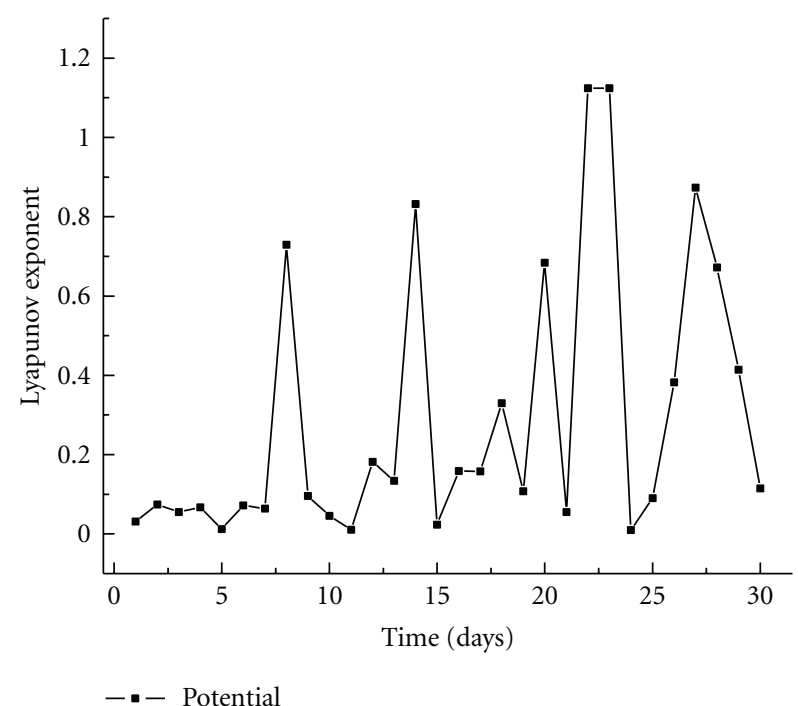

(a) EN potential

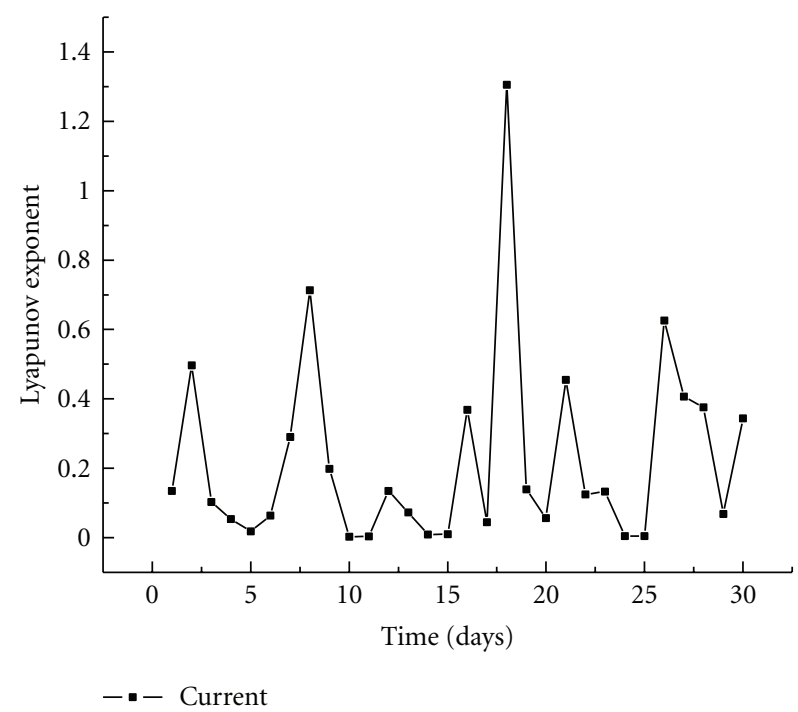

(b) EN current

Figure 7: Lyapunov exponents for EN time series as a function of time.

The observed behavior was modeled in terms of the circuit presented in Figure 7, a CPE in parallel with a charge transfer resistance and a second RC impedance element loop in parallel, corresponding to the low-frequency behaviour. When charge transfer is the control process, the circuit reduces to the classical equivalent Randles electric circuit of a charge transfer corrosion process [35, 37].

Sometimes impedance data obtained at the corrosion potential have the shape of a depressed semicircle with its centre, on the real axis. The simplest equivalent electric circuit corresponds to a parallel combination of a capacitance and a resistance. In the electric circuit, the constant phase element (CPE) represents the double-layer electrochemical

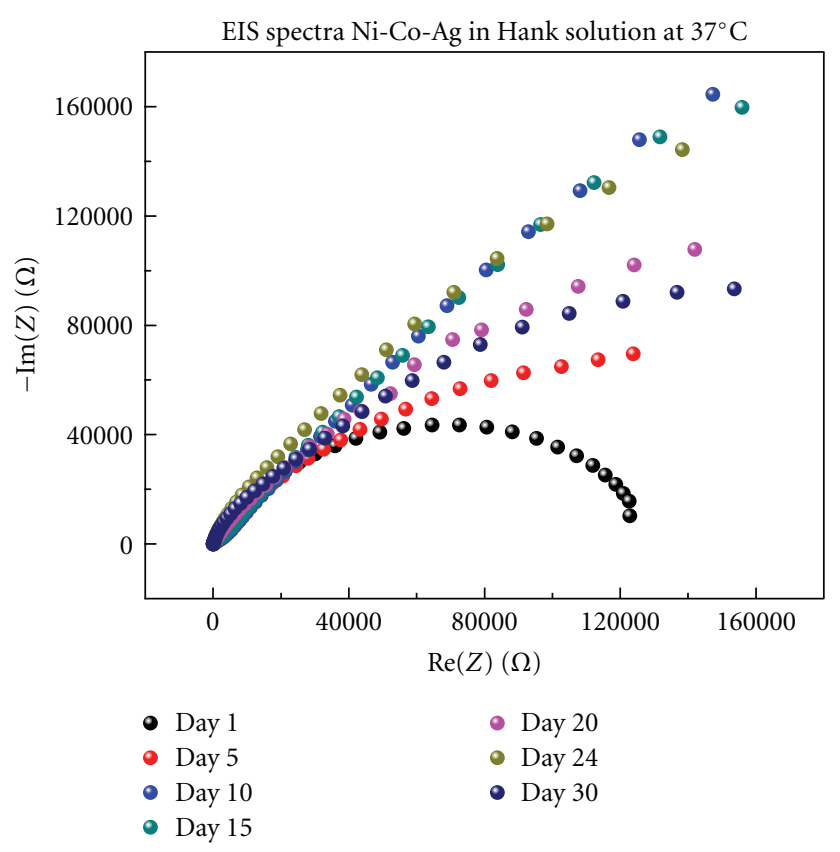

(a) Nyquist

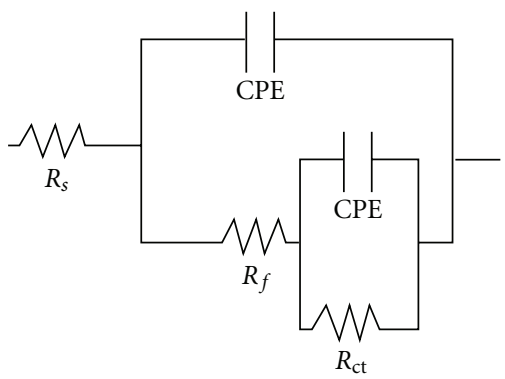

(b) Electric circuit

Figure 8: EIS Nyquist plot for different times of immersion and equivalent circuit.

interface, $R_{s}$ the solution resistance, and $R_{\mathrm{ct}}$ represents the charge transfer resistance.

The complex impedance $Z(j \omega)$ of a depressed semicircle could be expressed as

$$
Z=\frac{R_{s}+R_{\mathrm{ct}}}{\left[1+\left(j \omega C_{\mathrm{dl}} R_{\mathrm{ct}}\right)\right]^{n}} .
$$

For better quality constant phase elements, CPEs, replacing the capacitor, are often used in data fitting of depressed semicircles. The CPE is determined by the following equation $[38,39]$ :

$$
Z_{\mathrm{CPE}}=Z_{0}(j \omega)^{-n}=\frac{1}{Q(j \omega)^{n}},
$$

where $Z_{\mathrm{CPE}}$ is the $\mathrm{CPE}$ impedance; $Q$ corresponds to a proportionality factor, $j$ is $(-1)$; $\omega$ is the angular frequency and $n$ the surface irregularity estimation [40,41]. The CPE is considered to be a surface irregularity of the electrode [39], causing a depression in the Nyquist semicircle diagram [37]. 


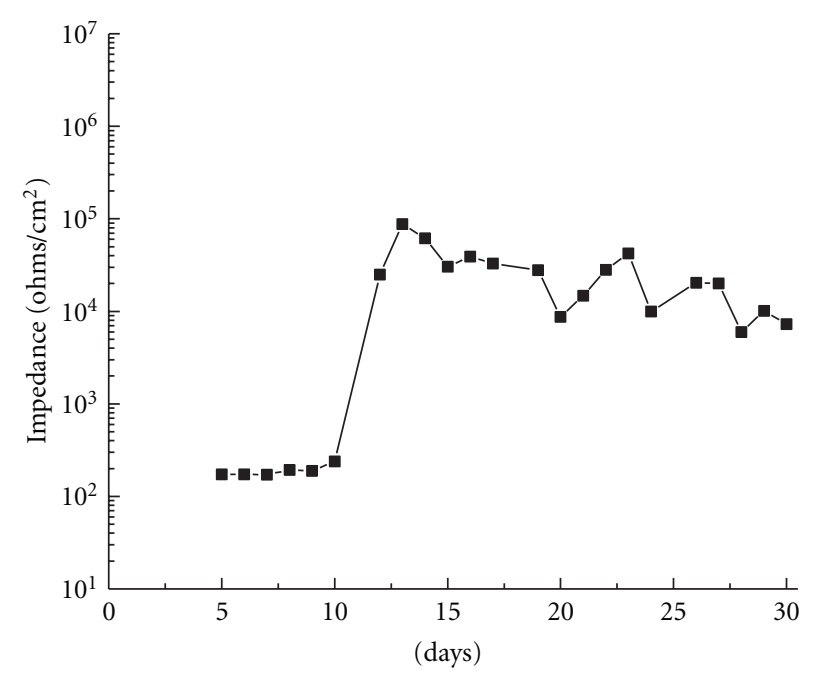

(a) Impedance

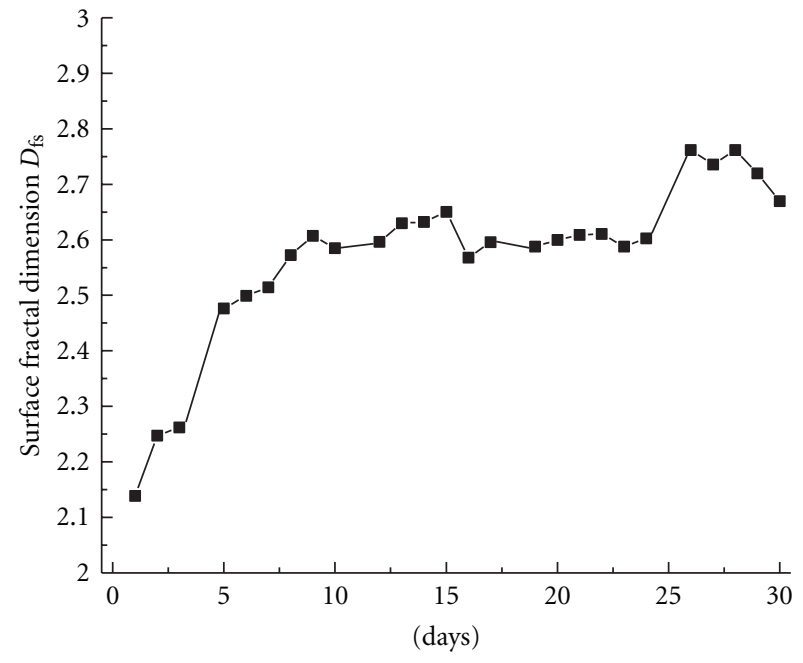

(b) Fractal Dimension

FIgURE 9: (a) Electrochemical impedance and (b) fractal dimension as a function of time.

The time constant $(\tau)$ and the capacitance value $(C)$ of the CPE elements can be calculated by the following $[42,43]$ :

$$
\begin{gathered}
Q=\frac{\tau^{n}}{R_{\mathrm{ct}}}, \\
C=\left[Q R_{\mathrm{ct}}^{(1-n)}\right]^{1 / n},
\end{gathered}
$$

where $\tau$ is the time constant and $C$ is the capacitance of the double layer associated with the CPE, with $\alpha$ being the depression angle:

$$
\alpha=(1-n) \times 90^{\circ} .
$$

Parameter $n$ is 1 for an ideal capacitor. In real systems, the ideal capacitive behaviour is hardly observed due to surface roughness, heterogeneities, or other effects that cause uneven current distributions over the electrode surface. In the case when $n=1$, the term $\left(j \omega C_{\mathrm{dl}} R_{\mathrm{ct}}\right)^{n}$, reduces to $j \omega C_{\mathrm{dl}} R_{\mathrm{ct}}$ where $C_{\mathrm{dl}}$ is the interfacial double-layer capacitance. This can be interpreted as an indication of the degree of heterogeneity of the metal surface [34]. When $n$ takes values slightly higher than 0.5 , it corresponds to a severe heterogeneity, but when $n$ is equal to 1 , the metal surface is completely smooth. This degree of heterogeneity has been associated to the fractal dimension of the surface [35].

A "fractal" is an object with complex structure, revealing new details at increasing degrees of magnification [29, 35]. Taking into account the degree of depression of the semicircle in the Nyquist impedance plot, it is possible to determine the fractal dimension (roughness) of the electrode surface by means of the following [44]:

$$
n=\frac{1}{\left(D_{\mathrm{fs}}-1\right)},
$$

where $D_{\mathrm{fs}}$ is the fractal dimension of the surface. $D_{\mathrm{fs}}$ can take values between 2 , for a surface completely smooth, to values close to 3, for a rough surface.

Generally, if the $D_{\mathrm{fs}}$ value falls around 2.0, this indicates that the metal surface is smooth and homogeneous; otherwise, if the value is lower than 3.0, it is because the metal surface is heterogeneous and rugged. Gasparovic et al. [45] explained the surface of an electrode by using the fractal geometry [33]. It has been demonstrated that the fractal dimension of an electrode can be determined by means of electrochemical impedance measurements and correlated with atomic force microscopy [35].

Electrochemical impedance as a function of time of immersion (Figure 9(a)) shows that in the first few days a low resistance was obtained, indicating that a high rate of corrosion occurred over the electrode surface; however, a steep increase in the impedance values due to a rapid growth of corrosion products layer over the metal surface probably occurred.

The surface fractal dimension $D_{\mathrm{fs}}$ ) as a function of time (Figure 9(b)) provides the connection with the impedance measurements (Figure 9(a)). It was observed that in the first few days of immersion, the fractal dimension of the electrode exhibits a low value, illustrating that the metallic surface starts homogeneously smooth. As time passes and the metal surface corrodes the fractal dimension increases, meaning that the surface is getting rough. After ten days of immersion, the fractal dimension reached a steady value $D_{\mathrm{fs}}$ 2.6), probably to the possible formation of a film of corrosion products, and the surface is expected to be homogeneously covered by the film. After 25 days, the fractal dimension reached the highest values probably due to further corrosion as indicated by the decreasing values of the impedance. Once again, further corrosion products grow or block the local site decreasing the fractal dimension again, to previous level. 
Nevertheless the value is still higher than that reported at the beginning of the test, idicating a highly rugged corroded surface as a consequence of the corrosion process.

The Nyquist impedance as a function of time presents an evolution from a charge transfer to a mass transfer corrosion process probably due to a film formation accompanied by eventual film rupture repassivation events, becoming a more complex process with rugged surface conditions. This is suggested by the complex structure of the phase space diagrams and the Lyapunov exponents and fractal dimension as a function of time obtained and presented.

\section{Conclusions}

Different EN time series were observed during the corrosion process for NiCoAg alloy in Hank solution, as a function of time of immersion. Corrosion proceeds as galvanic attack, followed by localized attack at silver particle sites, with corrosion products film formation. The dynamic corrosion of this alloy in solution is a chaotic process, according to fractal analysis of electrochemical noise. The fractal dimension obtained from electrochemical impedance is a measure of the corroded surface characterizing the attack. Chaotic analysis is a powerful tool to process electrochemical noise signals.

\section{References}

[1] J. B. D. Ratner, A. S. Hoffman, F. J. Schoen, and J. E. Lemon, Biomaterials Science: An Introduction to Materials in Medicine, Elsevier/Academic Press, New York, NY, USA, 2nd edition, 2004.

[2] M. J. Yaszemsk, Biomaterials in Orthopedics, Marcel Dekker, New York, NY, USA, 2004.

[3] J. M. Malo and J. Uruchurtu-Chavarin, "Electrochemical noise as a powerful electrchemical technique for corrosion studies," Research Trends, vol. 2, p. 49, 1997.

[4] D. A. Eden, "Electrochemical noise-the first two octaves," in Corrosion 98, NACE International, San Diego, Calif, USA, 1998.

[5] J. R. Kearns, J. R. Scully, P. R. Roberge, D. L. Reichert, and J. L. Dawson, Eds., Electrochemical Noise Measurement for Corrosion Applications, American Society for Testing and Materials, West Conshohocken, Pa, USA, 1996, STP 1277.

[6] R. A. Cottis, "Interpretation of electrochemical noise data," Corrosion, vol. 57, no. 3, pp. 265-284, 2001.

[7] A. Legat and V. Dolecek, "Chaotic analysis of electrochemical noise measured on stainless steel," Journal of the Electrochemical Society, vol. 142, no. 6, pp. 1851-1858, 1995.

[8] A. Legat, J. Osredkar, V. Kuhar, and M. Leban, "Detection of various types of corrosion processes by the chaotic analysis of electrochemical noise," Materials Science Forum, vol. 289-292, no. 2, pp. 807-812, 1998.

[9] J. Stringer and A. J. Markworth, "Applications of deterministic chaos theory to corrosion," Corrosion Science, vol. 35, no. 1-4, pp. 751-760, 1993.

[10] J. C. Uruchurtu and J. L. Dawson, "Noise analysis of pure aluminum under different pitting conditions," Corrosion, vol. 43, no. 1, pp. 19-26, 1987.

[11] A. J. Markworth, J. Stringer, and R. W. Rollins, "Deterministic chaos theory and its applications to materials science," $M R S$ Bulletin, vol. 20, no. 7, pp. 20-28, 1995.
[12] M. Keddam, M. Krarti, and C. Pallotta, "Some aspects of the fluctuations of the passive current on stainless steel in presence of chlorides-their relation to the probabilistic approach of pitting corrosion," Corrosion, vol. 43, no. 8, pp. 454-458, 1987.

[13] E. Rietman, Exploring the Geometry of Nature: Computer Modeling of Chaos, Fractals, Cellular Automata, and Neural Networks, Windcrest, 1988.

[14] J. T. Sandefur, Discrete Dynamical Systems: Theory and Applications, Clarendon Press, Ann Arbor, Mich, USA, 1990.

[15] E. García, J. Genescá, and J. Uruchurtu, "Pitting corrosion process of nickel in seawater. II. dynamic analysis," Afinidad, vol. 55, no. 473, pp. 26-30, 1998.

[16] "Effect of the electrolyte composition on the random/deterministic behaviors in pitting corrosion," in Proceedings of the Symposium on Critical Factors in Localized Corrosion III, S. Hoerle, T. Sourisseau, and B. Baroux, Eds., pp. 437-447, Electrochemical Society, Pennington, NJ, USA, 1999.

[17] in Proceedings of the Symposium Passivity and its Breakdown, S. Hoerle and B. Baroux, Eds., p. 57, Electrochemical Society, 1998.

[18] S. G. Corcoran and K. Sieradzki, "Chaos during the growth of an artificial pit," Journal of the Electrochemical Society, vol. 139, no. 6, pp. 1568-1573, 1992.

[19] "Chaotic behaviors in pitting corrosion processes," in Proceedings of the International Symposium on Pits and Pores: Formation, Properties, and Significance for Advanced Luminescent Materials, B. Baroux, H. Mayet, and D. Gorse, Eds., The Electrochemical Society, 1997.

[20] E. García, J. Uruchurtu, and J. Genescá, “Oscillatory dynamics of the pitting corrosion of copper in sea water," Afinidad, vol. 53, no. 464, pp. 215-222, 1996.

[21] U. Cano, J. Malo, and J. Uruchurtu, "Aluminium corrosion in chloride solution. is it chaos or is it electrochemical noise?" Corrosão e Protecção de Materiais, vol. 13, no. 1, pp. 6-12, 1994.

[22] P. R. Roberge, in Proceedings of the International Symposium Canadian Institute of Mining, p. 753, Canadian Institute of Mining, Metallurgy, and Petroleum, Canada, 1993.

[23] W. Li, K. Nobe, and A. J. Pearlstein, "Electrodissolution kinetics of iron in chloride solutions. VIII. Chaos in potential/current oscillations," Journal of the Electrochemical Society, vol. 140, no. 3, pp. 721-728, 1993.

[24] D. Sazou, M. Pagitsas, and C. Georgolios, "The influence of chloride ions on the dynamic characteristics observed at the transition between corrosion and passivation states of an iron electrode in sulphuric acid solutions," Electrochimica Acta, vol. 37, no. 11, pp. 2067-2076, 1992.

[25] Garcia, Uruchurtu, and Genesca, "Chaotic analysis of electrochemical noise response of copper," Anales de Química, vol. 94, no. 4-5, pp. 335-341, 1998.

[26] P. C. Searson and J. L. Dawson, "Analysis of electrochemical noise generated by corroding electrodes under open-circuit conditions," Journal of the Electrochemical Society, vol. 135, no. 8, pp. 1908-1915, 1988.

[27] H. C. Kuo and D. Landolt, "Galvanostatic transient study of anodic film formation on iron in concentrated chloride media," Corrosion Science, vol. 16, no. 12, pp. 915-922, 1976.

[28] W. Li, X. Wang, and K. Nobe, "Electrodissolution kinetics of iron in chloride solutions. VII. experimental potential/current oscillations," Journal of the Electrochemical Society, vol. 137, no. 4, pp. 1184-1188, 1990.

[29] T. Mulling, Ed., The Nature of Chaos, Oxford University Press, Oxford, UK, 1993.

[30] M. T. Rosenstein, J. J. Collins, and C. J. De Luca, "A practical method for calculating largest lyapunov exponents from small 
data sets," Physica D: Nonlinear Phenomena, vol. 65, no. 1-2, pp. 117-134, 1993.

[31] N. H. Packard, J. P. Crutchfield, J. D. Farmer, and R. S. Shaw, "Geometry from a time series," Physical Review Letters, vol. 45, no. 9, pp. 712-716, 1980.

[32] H. L. Swinney, "Observations of order and chaos in nonlinear systems," Physica D, vol. 7, no. 1-3, pp. 3-15, 1983.

[33] E. Sarmiento, J. G. González-Rodriguez, J. Uruchurtu, O. Sarmiento, and M. Menchaca, "Fractal analysis of the corrosion inhibition of carbon steel in a bromide solution by lithium chromate," International Journal of Electrochemical Science, vol. 4, no. 1, pp. 144-155, 2009.

[34] F. Barragán, R. Guardián, R. Menchaca, I. Rosales, and J. Uruchurtu, "Electrochemical corrosion of hot pressing titanium coated steels for biomaterial applications," International Journal of Electrochemical Science, vol. 5, pp. 1799-1809, 2010.

[35] J. A. Ruiz, I. Rosales, J. G. Gonzalez-Rodriguez, and J. Uruchurtu, "Effect of B on the corrosion resistance of a Ni-Ti alloy in simulated human body solution," International Journal of Electrochemical Science, vol. 5, no. 4, pp. 593-604, 2010.

[36] E. F. Diaz, J. G. Gonzalez-Rodriguez, R. Sandoval-Jabalera et al., "A study of the carboxyethylimidazoline as an $\mathrm{H}_{2} \mathrm{~S}$ corrosion inhibitor of X-120 pipeline steel," International Journal of Electrochemical Science, vol. 5, pp. 1821-1836, 2010.

[37] R. de Levie, "On the impedance of electrodes with rough interfaces," Journal of Electroanalytical Chemistry, vol. 261, no. 1, pp. 1-9, 1989.

[38] P. Zoltowski, "On the electrical capacitance of interfaces exhibiting constant phase element behaviour," Journal of Electroanalytical Chemistry, vol. 443, no. 1, pp. 149-154, 1998.

[39] D. A. Lopez, S. N. Simison, and S. R. de Sanchez, "The influence of steel microstructure on $\mathrm{CO}_{2}$ corrosion. EIS studies on the inhibition efficiency of benzimidazole," Electrochimica Acta, vol. 48, no. 7, pp. 845-854, 2003.

[40] F. Bentiss, M. Lebrini, and M. Lagrenee, "Thermodynamic characterization of metal dissolution and inhibitor adsorption processes in mild steel/2,5-bis(n-thienyl)-1,3,4-thiadiazoles/ hydrochloric acid system," Corrosion Science, vol. 47, no. 12, pp. 2915-2931, 2005.

[41] W. H. Li, Q. He, C. L. Pei, and B. R. Hou, "Experimental and theoretical investigation of the adsorption behaviour of new triazole derivatives as inhibitors for mild steel corrosion in acid media," Electrochimica Acta, vol. 52, no. 22, pp. 6386-6394, 2007.

[42] X. J. Wu, H. Y. Ma, S. H. Chen, Z. H. Xu, and A. F. Sui, "General equivalent circuits for faradaic electrode processes under electrochemical reaction control," Journal of the Electrochemical Society, vol. 146, no. 5, pp. 1847-1853, 1999.

[43] H. Y. Ma, X. L. Cheng, G. Q. Li et al., "The influence of hydrogen sulfide on corrosion of iron under different conditions," Corrosion Science, vol. 42, no. 10, pp. 1669-1683, 2000.

[44] D. Risovic, S. M. Poljacek, K. Furic, and M. Gojo, "Inferring fractal dimension of rough/porous surfaces-a comparison of SEM image analysis and electrochemical impedance spectroscopy methods," Applied Surface Science, vol. 255, no. 5, part 2, pp. 3063-3070, 2008.

[45] B. Gasparovic, D. Risovic, B. Cosovic, and A. Nelson, "The influence of frequency on fractal dimension of adsorbed layers," Electrochimica Acta, vol. 52, no. 7, pp. 2527-2534, 2007. 

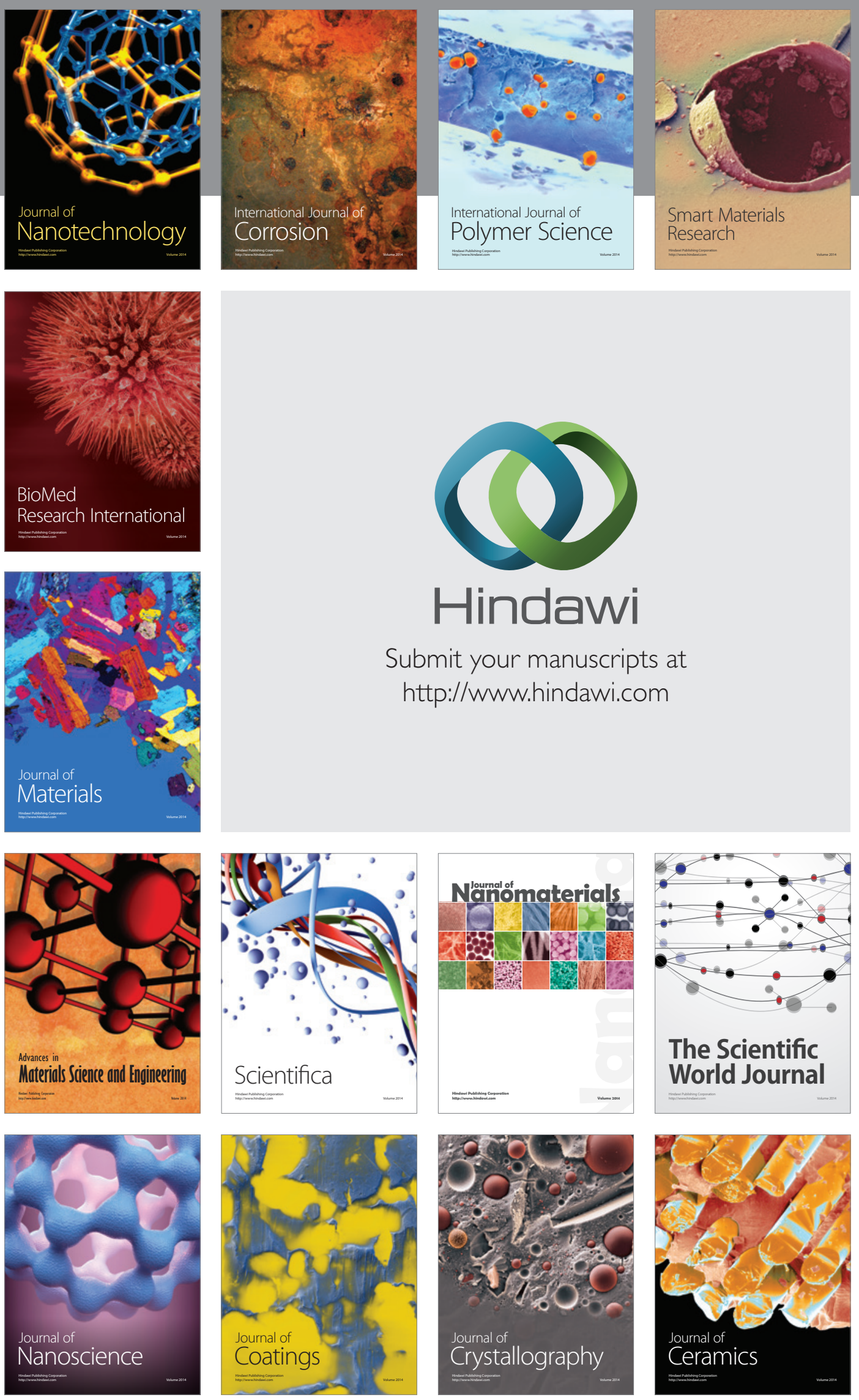

The Scientific World Journal

Submit your manuscripts at

http://www.hindawi.com

\section{World Journal}

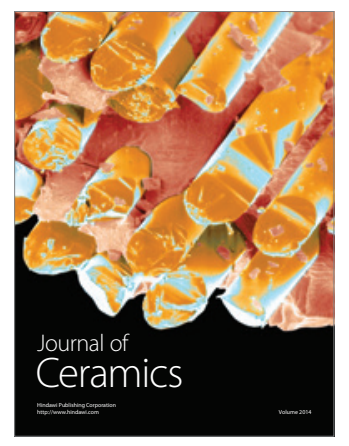

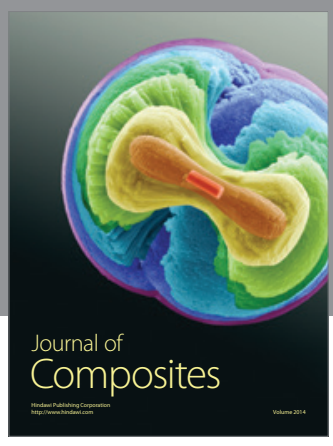
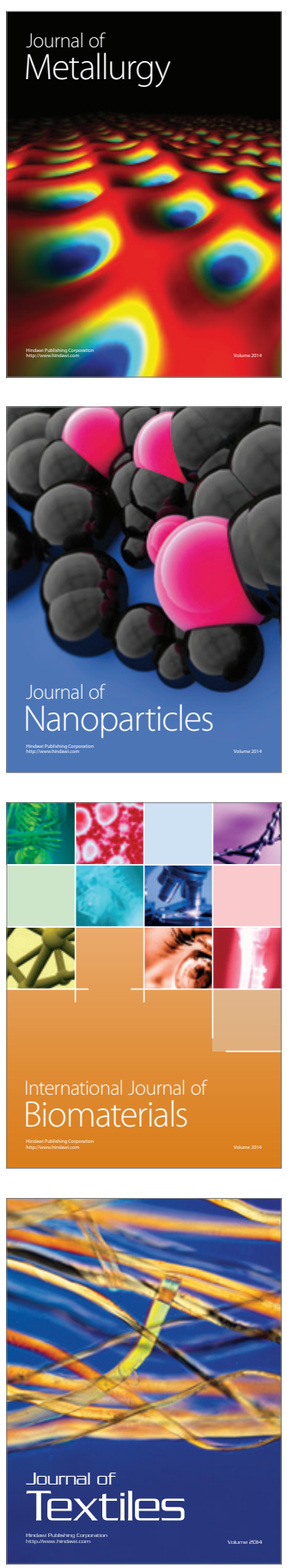